\title{
Modelo simple y generalizado para estimar la conductividad térmica de líquidos iónicos
}

\section{Simple and Generalized Model to Estimate the Thermal Conductivity of Ionic Liquids \\ Modelo simples e generalizado para estimar a condutividade térmica de líquidos iônicos}

\author{
Luis-Fernando Cardona-Palacio ${ }^{1}$
}

Recibido: mayo de 2021

Aceptado: agosto de 2021

Para citar este artículo: Cardona-Palacio, L. F. (2021). Modelo simple y generalizado para estimar la conductividad térmica de líquidos iónicos. Revista Científica, 42(3), 276-289. https://doi.org/10.14483/23448350.18052

\section{Resumen}

En este trabajo se generaliza un modelo empírico para predecir la conductividad térmica de líquidos iónicos. Inicialmente se realiza una recopilación de datos experimentales con diferentes tipos de aniones y cationes. En total, se evalúan 38 líquidos iónicos (28 sustancias para correlación y 10 sustancias para predicción) que corresponden a 265 datos experimentales. Los rangos de temperatura varían entre 273 K y $390 \mathrm{~K}$ a presión atmosférica (1 atm). Los parámetros del modelo son generalizados, utilizando 215 datos experimentales y las desviaciones promedio son de $4.72 \%$. Posteriormente, se evalúan las capacidades predictivas en 50 datos experimentales con desviaciones promedio de $3.48 \%$. El modelo generalizado es extendido a mezclas binarias utilizando una regla de mezcla simple con un parámetro de interacción y la desviación absoluta es de 0.77 $\%$. Finalmente, el modelo es comparado con otros en la literatura y los resultados estadísticos muestran que proporciona resultados aceptables.
Palabras clave: conductividad térmica; correlación; líquidos iónicos; mezclas; modelo empírico; predicción.

\begin{abstract}
In this work, an empirical model is generalized to predict the thermal conductivity of ionic liquids. Initially, experimental data are collected, which include different types of anions and cations. A total of 38 ionic liquids are evaluated (28 substances for correlation and 10 substances for prediction), which corresponds to 265 experimental data. The temperature ranges vary between 273 and $390 \mathrm{~K}$ at atmospheric pressure $(1 \mathrm{~atm})$. The model is generalized using 215 experimental data, and the average deviation is around $4.72 \%$. Then, the predictive capabilities are evaluated in 50 experimental data with an average deviation of $3.48 \%$. The generalized model is extended to binary mixtures using a simple mixing rule with an interaction parameter, and the absolute deviation is $0.77 \%$. Finally, the generalized model
\end{abstract}

1. Ph. D. Universidad Católica Luis Amigó, Medellín, Colombia. luis.cardonapa@amigo.edu.co. 
is compared with others developed in the literature, and the statistical analysis shows that it provides acceptable results.

Keywords: correlation; empirical model; ionic liquids; mixtures; prediction; thermal conductivity.

\section{Resumo}

Neste trabalho é generalizado um modelo empírico para prever a condutividade térmica de líquidos iônicos. Inicialmente, é realizada uma compilação de dados experimentais com diferentes tipos de ânions e cátions. No total, 38 líquidos iônicos são avaliados (28 substâncias para correlação e 10 substâncias para previsão) correspondendo a 265 dados experimentais. As faixas de temperatura variam entre $273 \mathrm{~K}$ e $390 \mathrm{~K}$ à pressão atmosférica (1 atm). Parâmetros do modelo generalizado são generalizadas usando 215 dados experimentais e os desviações promédios são 4.72 \%. Posteriormente, as capacidades preditivas são avaliadas em 50 dados experimentais com desviações promédios de $3.48 \%$. O modelo generalizado é estendido para misturas binárias usando uma regra de mistura simples com um parâmetro de interação e o desviações promédios é 0,77 \%. Por último, o modelo é comparado com outros da literatura e os resultados estatísticos mostram que ele fornece resultados aceitáveis.

Palavras-chaves: condutividade térmica; correlação; líquidos iônicos; misturas; modelo empírico; predição.

\section{Introducción}

Los líquidos iónicos (LIs) son sales formadas por aniones y cationes y se caracterizan por tener un bajo de punto de fusión (Alvarez-Guerra et al., 2011). Estos líquidos presentan unas propiedades fisicoquímicas que son de interés en la comunidad científica e industrial. Estas propiedades corresponden a una presión de vapor prácticamente despreciable, una alta estabilidad térmica, altas viscosidades en comparación con disolventes orgánicos, una conductividad térmica casi constante, entre otras (Castillo, 2015; Alvarez-Guerra et al., 2011).

En particular, la conductividad térmica es una propiedad de transporte útil para diseñar equipos de ingeniería como intercambiadores de calor, equipos de agitación y mezcla, entre otros (Díaz-Parra y Vera-López, 2018). Recientemente, la estimación de esta propiedad ha posibilitado diseñar equipos que involucran nanofluidos (Ranjbarzadeh et al., 2019). Cuando se carece de datos experimentales, esta importante propiedad debe ser estimada utilizando modelos empíricos, teóricos o semi-teóricos (Bonyadi y Rostami, 2017) y los basados en redes neuronales e inteligencia artificial (Hezave et al., 2012). Ejemplo de estos modelos son los desarrollados por Hopp y Gross (2019), Cardona et al. (2019), Tomassetti et al. (2020), Chen et al. (2020). No obstante, estos modelos han sido utilizados para predecir sustancias orgánicas y no se han extendido a líquidos iónicos. Diferentes modelos han sido propuestos para realizar estimaciones de la conductividad térmica de líquidos iónicos, entre los que se destacan los trabajos realizados por Frez et al. (2006), Ge et al. (2007), Gardas y Coutinho (2009), Hezave et al. (2012), Cardona y Valderrama (2020a). Estos modelos tienen características empíricas o están basados en redes neuronales artificiales o modelos teóricos.

Los modelos empíricos se basan en el comportamiento experimental en términos de la temperatura. Las representaciones matemáticas de estos modelos son funciones lineales en términos de la temperatura (Ge et al. (2007)) o por funciones no lineales en términos de la densidad y la capacidad calorífica (Fröba et al., 2010) o utilizando técnicas de contribución de grupos (Gardas y Coutinho (2009)). Recientemente se han utilizado los métodos basados en el concepto de similitud geométrica mediante la comparación de dos diagramas termodinámicos como P $\rho \mathrm{T}$ y $\mathrm{P} \lambda \mathrm{T}$. Ejemplo de este tipo de modelo es el propuesto por Cardona y Valderrama (2020a) que utiliza la ecuación de estado de Patel-Teja-Valderrama generalizada en términos del peso molecular con desviaciones promedio de $15.6 \%$.

Por otro lado, diferentes modelos basados en redes neuronales artificiales (ANN) han sido 
desarrollados en la literatura (Hezave et al., 2012; Akhgar et al., 2019). El trabajo desarrollado por Hezave et al. (2012) es uno de los más representativos de esta línea de investigación. Los autores utilizaron un único número de neuronas ocultas y 13 en capa oculta con una función del tipo sigmoidal logarítmica y purelin como las funciones de transferencia en las capas ocultas y de salida. Las desviaciones absolutas relativas promedio varían entre 0.20 \% y 2.7 \% para 20 líquidos iónicos; sin embargo, no se reportan los valores máximos de desviación.

Finalmente, el modelo teórico propuesto por Frez et al. (2006) depende de la densidad y la capacidad calorífica y presenta desviaciones que varían entre $1.9 \%$ y $24.8 \%$ cuando se aplica para predecir cuatro líquidos iónicos del tipo imidazolium.

La mayoría de los modelos descritos en los párrafos anteriores no están generalizados (Ge et al. (2007)), no se reportan variables estadísticas que demuestren la robustez y bondad de un modelo (Hezave et al. (2012)) y son utilizados para un grupo reducido de líquidos iónicos (Gardas y Coutinho 2009). Por estos motivos, en el presente trabajo se generaliza un modelo empírico que logre realizar predicciones de la conductividad térmica en un número representativo de líquidos iónicos y sea utilizado en procesos industriales como refrigeración y calefacción (Asensio-Delgado et al., 2020).

\section{Modelo de conductividad térmica y metología}

A continuación se exponen el modelo de conductividad térmica y la metodología propuesta en la presente investigación.

\section{Modelo de conductividad térmica}

El modelo empírico desarrollado por Chen et al. (2020) ha sido utilizado para realizar predicciones de la conductividad térmica en refrigerantes y en este trabajo es extendido a líquidos iónicos con la ecuación 1:

$$
\lambda=\frac{a+b T_{r}}{\left(c+T_{r}\right)^{d}}
$$

donde, $\lambda$ es la conductividad térmica en $\mathrm{W} / \mathrm{mK}$, $T_{r}$ es la temperatura reducida estimada como $T_{r}=$ $\mathrm{T} / \mathrm{T}_{\mathrm{c}}$ ( $\mathrm{T}$ es la temperatura en Kelvin, $\mathrm{T}_{\mathrm{c}}$ es la temperatura crítica en Kelvin y $T_{r}$ es la temperatura reducida, adimensional) y $a, b, c$ y $d$ son parámetros que dependen de cada sustancia. Para la estimación de la temperatura crítica se utiliza en este trabajo la metodología propuesta por Valderrama et al. (2019a). Esta metodología estima las propiedades críticas $\left(T_{c^{\prime}} P_{c^{\prime}}, V_{c}\right)$ y el factor acéntrico a partir de técnicas de contribución de grupos de un conjunto representativo de líquidos iónicos. El modelo empírico de conductividad térmica es consistente en unidades donde a y $b$ están dados en $\mathrm{W} / \mathrm{mK}$, y c y $d$ son adimensionales.

\section{Selección de datos experimentales}

El modelo de conductividad térmica requiere de cuatro parámetros por líquido iónico $(a, b, c, d)$ que deben ser determinados utilizando datos experimentales. Todos los datos experimentales son obtenidos a partir de los valores recopilados por la base de datos de la NIST-LIs (Dong et al., 2007; Kazakov et al., s. f.; Cardona y Valderrama (2020a)). Estos datos son clasificados en tres categorías: 1) un primer conjunto lo conforman aquellos obtenidos para el proceso de correlación y generalización; 2) un segundo conjunto lo conforman aquellos obtenidos para el proceso de predicción y 3) un tercer grupo lo conforman aquellos utilizados para analizar la extensión del modelo a mezclas binarias conformadas por soluciones acuosas (agua+líquido iónico). Las incertidumbres estimadas por la NIST-LIs varían entre un $1 \%$ y $14 \%$. Estos valores de incertidumbres son obtenidos a partir de la información recopilada por cada trabajo reportado en la literatura y se estiman a partir de los materiales y métodos, el tipo de dato y el proceso de experimentación en cuanto a las incertidumbres de los equipos de medición (Dong et al., 
2007; Bloxham et al., 2020; Kazakov et al., s. f.). Es importante aclarar que las sustancias utilizadas en el proceso de correlación y predicción son seleccionadas al azar mediante la función aleatorio de MS Excel.

En la tabla 1 se muestra un resumen de las propiedades físicas (velocidad del sonido, tensión superficial) y de transporte (viscosidad y conductividad térmica) de los líquidos iónicos utilizados en este trabajo. Los rangos de los valores de estas propiedades son obtenidos a partir de los datos experimentales recopilados por la NIST-LIs. En la columna 1 de la tabla 1 se encuentra el tipo de catión que conforma el líquido iónico, en la columna 2 el número de líquidos iónicos trabajados en este estudio y las columnas 3 a 7 representan los rangos de temperatura, conductividad térmica $(\lambda)$, viscosidad $(\mu)$, tensión superficial $(\sigma)$ y velocidad del sonido (s). Estas propiedades son utilizadas en el diseño y optimización de procesos industriales (Poling et al., 2001).

En la tabla 1, el $79 \%$ de los líquidos iónicos son del tipo imidazolium, seguido de pyridinium con un $11 \%$ y finalizando con phosphonium (5 $\%$ ), pyrrolidinium y ammonium (3\%). Frente a las propiedades físicas y de transporte, se observa que la conductividad térmica varía entre $1.17 \cdot 10$ ${ }^{1}$ y $2.04 \cdot 10^{-1} \mathrm{~W} / \mathrm{mK}$, la velocidad del sonido varía entre $1.17 \cdot 10^{3}$ y $2.89 \cdot 10^{3} \mathrm{~m} / \mathrm{s}$ y la tensión superficial entre $2.15 \cdot 10^{-2}$ y $5.04 \cdot 10^{-2} \mathrm{~N} / \mathrm{m}$, valores que se encuentran dentro de lo reportado por diferentes trabajos en la literatura (Valderrama et al., 2019b; Cardona y Valderrama (2020a) y Cardona y Valderrama (2020b)). En particular, la viscosidad de los líquidos iónicos varía entre $1.10 \cdot 10^{-2}$ y $8.54 \cdot 10^{-1}$ $\mathrm{Pa} \cdot \mathrm{s}$, altos valores cuando son comparados con el agua $\left(\mu=1.00 \cdot 10^{-3} \mathrm{~Pa} \cdot \mathrm{s}\right.$ a $\mathrm{T}=293 \mathrm{~K}$ y $\left.\mathrm{P}=1 \mathrm{~atm}\right) \mathrm{y}$ el decano $\left(\mu=9.16 \cdot 10^{-4} \mathrm{~Pa} \cdot \mathrm{s}\right.$ a $\mathrm{T}=293 \mathrm{~K}$ y $\mathrm{P}=1$ atm). Esta versatilidad de las propiedades físicas y de transporte genera que los líquidos iónicos, mediante la combinación de un anión y un catión, puedan utilizarse en diferentes aplicaciones como en la industria de solventes, aditivos de combustibles y lubricantes, procesos de separación, materiales electrostáticos, entre otros (Minea, 2020).

\section{Determinación de parámetros}

Los parámetros óptimos del modelo de conductividad térmica son determinados a partir de datos experimentales y mediante regresión no lineal, empleando el algoritmo de Lasdon et al. (1978). Este algoritmo ha sido empleado para resolver problemas de optimización en áreas del conocimiento como la biotecnología (Kemmer y Keller, 2010; Bhunia y Ghangrekar, 2008), la planeación de producción (Burke, 2016), la docencia (Godsen, 2002; Iglesias et al., 2004; Rivas et al., 2006), el diseño de productos y procesos (Huang et al., 2009; Cardona, 2016), entre otras. Este también

Tabla 1. Rangos de temperatura y propiedades físicas y de transporte de los líquidos iónicos analizados en el presente trabajo

\begin{tabular}{|c|c|c|c|c|c|c|}
\hline catión & $\begin{array}{c}\text { No. Líquidos } \\
\text { iónicos }\end{array}$ & $\Delta \mathbf{T}(\mathbf{K})$ & $\Delta \lambda(\mathbf{W} / \mathbf{m K})$ & $\Delta \mu(\mathrm{Pa} \cdot \mathrm{s})$ & $\Delta \sigma(\mathrm{N} / \mathrm{m})$ & $\Delta \mathrm{s}(\mathrm{m} / \mathrm{s})$ \\
\hline imidazolium & 30 & $\begin{array}{c}273.15 \\
-390 \\
\end{array}$ & $\begin{array}{r}1.19 \cdot 10^{-1} \\
-2.04 \cdot 10^{-1} \\
\end{array}$ & $\begin{array}{l}1.22 \cdot 10^{-2} \\
-7.66 \cdot 10^{-1} \\
\end{array}$ & $\begin{array}{r}2.15 \cdot 10^{-2} \\
-5.04 \cdot 10^{-2} \\
\end{array}$ & $\begin{array}{r}1.17 \cdot 10^{3} \\
-2.89 \cdot 10^{3} \\
\end{array}$ \\
\hline ammonium & 1 & $\begin{array}{r}273.15 \\
-353.15 \\
\end{array}$ & $\begin{array}{r}1.18 \cdot 10^{-1} \\
-1.21 \cdot 10^{-1} \\
\end{array}$ & $\begin{array}{r}1.10 \cdot 10^{-2} \\
-2.38 \cdot 10^{-1} \\
\end{array}$ & $\begin{array}{c}3.50 \cdot 10^{-2} \\
-3.67 \cdot 10^{-2} \\
\end{array}$ & $\begin{array}{r}1.45 \cdot 10^{3} \\
-1.55 \cdot 10^{3} \\
\end{array}$ \\
\hline pyrrolidinium & 1 & $\begin{array}{c}293 \\
-323\end{array}$ & $\begin{array}{c}1.28 \cdot 10^{-1} \\
-1.88 \cdot 10^{-1}\end{array}$ & $\begin{array}{c}4.20 \cdot 10^{-3} \\
-9.86 \cdot 10^{-2}\end{array}$ & $\begin{array}{l}5.08 \cdot 10^{-2} \\
-5.64 \cdot 10^{-2}\end{array}$ & $\begin{array}{r}1.70 \cdot 10^{3} \\
-1.84 \cdot 10^{3}\end{array}$ \\
\hline pyridinium & 4 & $\begin{array}{l}294.2 \\
-375 \\
\end{array}$ & $\begin{array}{r}1.17 \cdot 10^{-1} \\
-1.74 \cdot 10^{-1} \\
\end{array}$ & $\begin{array}{r}1.70 \cdot 10^{-2} \\
-7.01 \cdot 10^{-1} \\
\end{array}$ & $\begin{array}{l}4.42 \cdot 10^{-2} \\
-4.79 \cdot 10^{-2} \\
\end{array}$ & $\begin{array}{r}1.51 \cdot 10^{3} \\
-1.65 \cdot 10^{3} \\
\end{array}$ \\
\hline phosphonium & 2 & $\begin{array}{c}282.47 \\
-353.62\end{array}$ & $\begin{array}{c}1.35 \cdot 10^{-1} \\
-1.40 \cdot 10^{-1}\end{array}$ & $\begin{array}{c}2.72 \cdot 10^{-2} \\
-8.54 \cdot 10^{-1}\end{array}$ & $\begin{array}{c}2.75 \cdot 10^{-2} \\
-3.36 \cdot 10^{-2}\end{array}$ & $\begin{array}{c}1.39 \cdot 10^{3} \\
-1.60 \cdot 10^{3}\end{array}$ \\
\hline
\end{tabular}


es llamado el método del gradiente reducido generalizado (GRG). Los parámetros óptimos corresponden a aquellos que minimizan el porcentaje de desviación absoluta relativa promedio de la conductividad térmica (AAD $\lambda$ ). La función objetivo a minimizar es ampliamente utilizada en diferentes investigaciones en la determinación de parámetros de modelos termodinámicos (Cardona y Valderrama (2020a) y 2020b) y utilizando la ecuación 2:

$$
\text { F. O. }=\operatorname{AAD} \lambda(\overrightarrow{\mathrm{X}})=\frac{100}{\mathrm{~N}} \sum_{\mathrm{i}=1}^{\mathrm{N}}\left|\frac{\lambda_{\mathrm{cal}}(\overrightarrow{\mathrm{X}})-\lambda_{\exp }}{\lambda_{\exp }}\right|
$$

Considerando que $\overrightarrow{\mathrm{x}}=\{a, b, c, d\}$. Donde, $\mathrm{AAD} \lambda$ es la desviación absoluta relativa promedio entre los valores predichos por el modelo y los valores experimentales, $\lambda_{\text {exp }}$ y $\lambda_{\text {cal }}$ representan las conductividades térmicas experimental y calculada y $N$ es el número de datos ( $\lambda$ es la conductividad térmica en $\mathrm{W} / \mathrm{mK})$. El vector $X$ corresponde al conjunto de parámetros que deben ser estimados para cada líquido iónico. Adicionalmente, se calculan desviaciones relativas promedio (AD $\lambda$ ) y valores máximos de desviación (MD $\lambda$ ) de cada líquido iónico de acuerdo con las ecuaciones 3 y 4 :

$$
\begin{aligned}
& \mathrm{AD} \lambda=\frac{100}{\mathrm{~N}} \sum_{\mathrm{i}=1}^{\mathrm{N}}\left(\frac{\lambda_{\mathrm{cal}}-\lambda_{\text {exp }}}{\lambda_{\text {exp }}}\right) \\
& \mathrm{MD} \lambda=\text { valor máx.de } 100 \cdot\left|\frac{\lambda_{\text {cal }}-\lambda_{\text {exp }}}{\lambda_{\text {exp }}}\right|
\end{aligned}
$$

Las ecuaciones 3 y 4 representan variables estadísticas básicas que establecen la bondad y robustez de un modelo (Valderrama y Álvarez, 2005). Es importante aclarar que el modelo generalizado desarrollado en este trabajo tiene características empíricas.

\section{Proceso de generalización}

Con el conjunto de parámetros óptimos de cada líquido iónico estimados anteriormente, se analiza el comportamiento grafico en términos del peso molecular (Daubert, 1998). Lo anterior, permite proponer una expresión matemática para cada parámetro y mediante un proceso de minimización utilizando la ecuación 2 se estiman las constantes características del modelo.

\section{Extensión del modelo a mezclas}

El modelo generalizado de conductividad térmica es extendido a mezclas binarias utilizando la regla de mezcla simple propuesta por Huang et al. (2015) y la expresión matemática se ilustra en las ecuaciones 5 y 6 .

$$
\begin{aligned}
& \lambda_{\mathrm{m}}=\mathrm{x}_{1} \lambda_{1}+\mathrm{x}_{2} \lambda_{2}+\delta \lambda \\
& \delta \lambda=\pi \mathrm{x}_{1} \mathrm{x}_{2}\left(\lambda_{1}+\lambda_{2}\right) \mathrm{T}^{1 / 2}
\end{aligned}
$$

Donde $\lambda_{\mathrm{m}}$ es la conductividad térmica de la mezcla, $x_{1}$ y $x_{2}$ son las fracciones molares de cada componente puro y $\pi$ es un parámetro de interacción binaria que depende de cada mezcla y debe ser estimado utilizando datos experimentales. Es importante anotar que la ecuación 5 se compone de dos términos que representan lo ideal y no ideal (Huang et al. (2015)).

\section{Resultados y discusión}

A continuación, se presentan los resultados obtenidos para el proceso de correlación, generalización, predicción y mezclas binarias.

\section{Resultados del proceso de correlación y generalización}

En total, 215 datos experimentales que representan 28 de líquidos iónicos son utilizados durante la correlación y generalización. Es importante aclarar que el conjunto de datos experimentales es obtenido a partir de los valores reportados por diferentes autores en la literatura y compilados en la base de datos de la NIST-LIs (Dong et al., 2007; Kazakov et 
al., s. f.; Cardona y Valderrama (2020a)). En la tabla 2 se muestra el conjunto de sustancias utilizadas en estos procesos, en la columna 2 se encuentra el líquido iónico, mientras que en las columnas de 3 a 7 están los valores del número de datos $(\mathrm{N})$ : peso molecular $(\mathrm{M})$, temperatura crítica $\left(\mathrm{T}_{c}\right)$, rangos de temperatura $(\Delta \mathrm{T})$ y conductividad térmica $(\Delta \lambda)$. La nomenclatura de identificación de cada líquido iónico (columna 2) se da a partir de lo reportado por Valderrama et al. (2019a). Inicialmente se estiman para cada líquido iónico los valores de los parámetros óptimos utilizando el proceso de minimización dado por la ecuación 2.

En la figura 1 se muestran los valores óptimos de los parámetros $(a, b, c$ y $d$ ) en términos del peso molecular. En esta figura, el parámetro a exhibe una clara tendencia cuadrática en términos del peso molecular ( $\mathrm{M}$ en $\mathrm{g} / \mathrm{mol})$. Por otro lado, los parámetros $b$, c y $d$ tienen un comportamiento constante (ver figura 1). Es importante anotar que el peso molecular es un descriptor molecular fácil de estimar y es utilizado en aplicaciones de generalización de modelos de transporte (viscosidad y conductividad térmica), al igual que en modelos de tensión superficial (Poling et al., 2001; Cardona y Valderrama 2020a y 2020b).

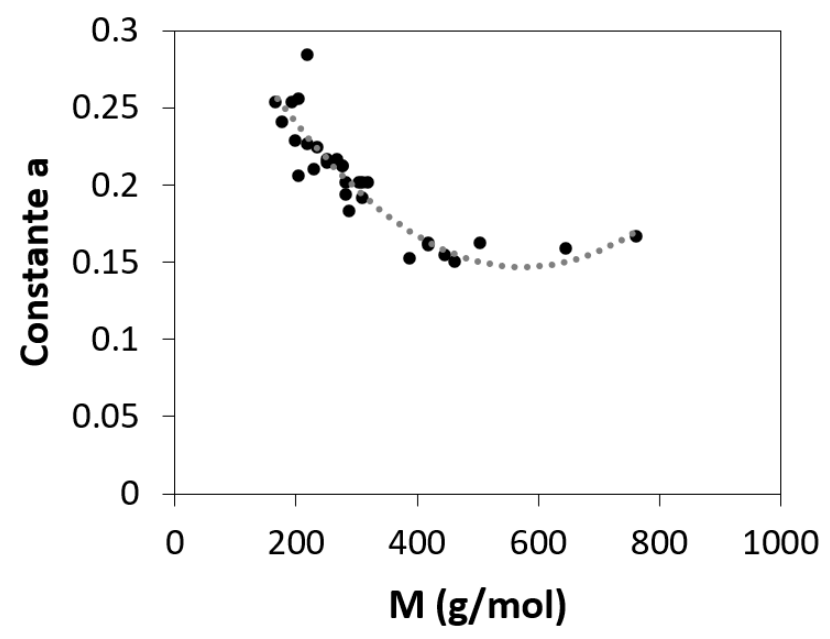

- Constante a
Posteriormente, se realiza el proceso de optimización utilizando la función objetivo a minimizar usando la ecuación 2 y tomando $a=\alpha \cdot M^{2}+\beta \cdot M+$ $\gamma, b, c$ y $d$ constantes para todos los líquidos iónicos. En la tabla 2 se muestran las desviaciones obtenidas en el proceso de generalización (columnas 8 a 10) y en la tabla 3 se ilustra el modelo generalizado con las constantes óptimas.

En general, las desviaciones relativas absolutas promedio obtenidas en el proceso de generalización $(A A D \lambda)$ son de $4.72 \%(M D \lambda=5.96 \%)$, y las desviaciones relativas promedio (AD $\lambda$ ) son de $-0.83 \%$. En la tabla 4 se muestra la capacidad de generalización del modelo para describir los datos experimentales reportados por la NIST-LIs (Dong et al., 2007; Kazakov et al., s. f.). Los resultados presentados en la figura 1 y tabla 4 muestran las apropiadas capacidades del modelo para describir la conductividad térmica con bajas desviaciones que se encuentran dentro de la incertidumbre experimental.

\section{Resultados del proceso de predicción}

Se evalúa la capacidad del modelo en describir un conjunto de líquidos iónicos diferentes a los

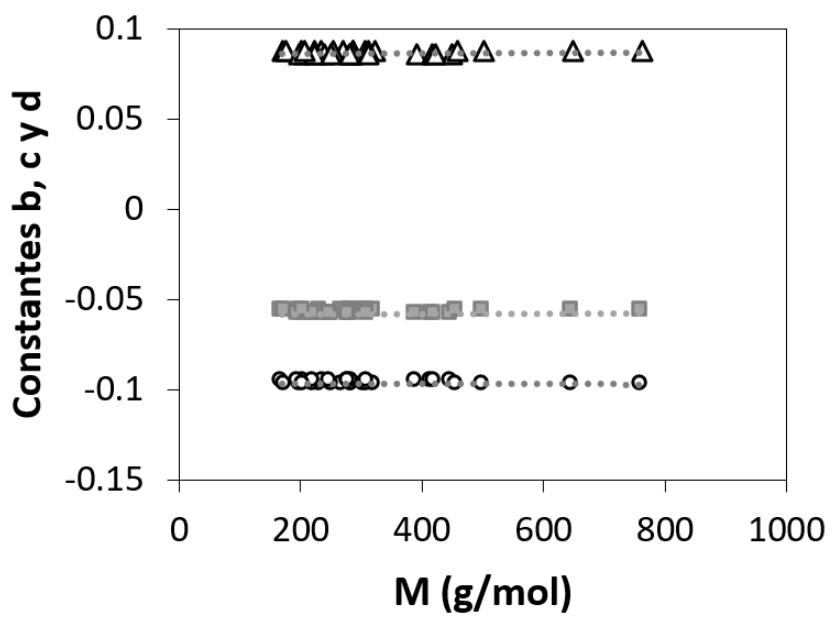

○ Constante b $\Delta$ Constante c $\square$ Constante d

Figura 1. Comportamiento de los parámetros en términos del peso molecular.

Fuente: elaboración propia 
Tabla 2. Rangos de temperatura, conductividad térmica y desviaciones durante el proceso de generalización

\begin{tabular}{|c|c|c|c|c|c|c|c|c|c|}
\hline No. & Líquido iónico & $\mathbf{N}$ & $\mathrm{M}(\mathrm{g} / \mathrm{mol})$ & $T_{c}(K)$ & $\Delta \mathbf{T}(\mathrm{K})$ & $\Delta \lambda(\mathbf{W} / \mathrm{mK})$ & $\mathrm{AD} \lambda$ & $\mathrm{AAD} \lambda$ & $\mathrm{MD} \lambda$ \\
\hline 1 & {$[\mathrm{C} 2 \mathrm{mim}][\mathrm{Ac}]$} & 9 & 170.21 & 807.14 & $273.15-353.15$ & $\begin{array}{c}1.99 \cdot 10^{-1} \\
-2.04 \cdot 10^{-1} \\
\end{array}$ & -2.03 & 2.96 & 4.92 \\
\hline 2 & [C6mim] [B(CN)4] & 8 & 234.12 & 1084.16 & $283.15-353.15$ & $\begin{array}{c}1.78 \cdot 10^{-1} \\
-1.81 \cdot 10^{-1}\end{array}$ & 4.39 & 4.39 & 7.14 \\
\hline 3 & {$[\mathrm{C} 10 \mathrm{mim}][\mathrm{B}(\mathrm{CN}) 4]$} & 8 & 304.25 & 1186.02 & $283.15-353.15$ & $\begin{array}{c}1.55 \cdot 10^{-1} \\
-1.58 \cdot 10^{-1} \\
\end{array}$ & -4.33 & 4.33 & 5.83 \\
\hline 4 & [C2mim] [C8SO4] & 9 & 320.46 & 1157.12 & $273.15-353.15$ & $\begin{array}{c}1.50 \cdot 10^{-1} \\
-1.54 \cdot 10^{-1} \\
\end{array}$ & -8.53 & 8.53 & 10.61 \\
\hline 5 & [C2mim] [dca] & 9 & 177.21 & 998.96 & $273.15-353.15$ & $\begin{array}{c}2.00 \cdot 10^{-1} \\
-2.04 \cdot 10^{-1}\end{array}$ & 1.34 & 1.89 & 4.15 \\
\hline 6 & [C2mim] [ESO4] & 24 & 236.29 & 1067.49 & $273.15-353.15$ & $\begin{array}{c}1.77 \cdot 10^{-1} \\
-1.80 \cdot 10^{-1} \\
\end{array}$ & -2.87 & 2.87 & 4.61 \\
\hline 7 & [C2mim] [mesy] & 2 & 206.27 & 1026.03 & $300-375$ & $\begin{array}{c}1.87 \cdot 10^{-1} \\
-1.90 \cdot 10^{-1}\end{array}$ & -10.4 & 10.4 & 11.25 \\
\hline 8 & {$[\mathrm{C} 2 \mathrm{mim}][\mathrm{tcc}]$} & 9 & 201.23 & 1149.35 & $273.15-353.15$ & $\begin{array}{c}1.92 \cdot 10^{-1} \\
-1.95 \cdot 10^{-1}\end{array}$ & 1.79 & 2.11 & 5.42 \\
\hline 9 & [C6mim] [tcc] & 8 & 271.37 & 1241.91 & $283.15-353.15$ & $\begin{array}{c}1.67 \cdot 10^{-1} \\
-1.69 \cdot 10^{-1} \\
\end{array}$ & -7.04 & 7.04 & 8.2 \\
\hline 10 & [C8mim] [tcc] & 8 & 285.40 & 1261.82 & $283.15-353.15$ & $\begin{array}{c}1.62 \cdot 10^{-1} \\
-1.65 \cdot 10^{-1}\end{array}$ & -2.46 & 2.46 & 4.25 \\
\hline 11 & [C10mim] [tcc] & 8 & 313.45 & 1303.26 & $283.15-353.15$ & $\begin{array}{c}1.54 \cdot 10^{-1} \\
-1.57 \cdot 10^{-1}\end{array}$ & -6.45 & 6.45 & 7.98 \\
\hline 12 & [C4mim] [TfO] & 7 & 288.29 & 1023.54 & $293-353$ & $\begin{array}{c}1.58 \cdot 10^{-1} \\
-1.61 \cdot 10^{-1}\end{array}$ & 10.12 & 10.12 & 10.91 \\
\hline 13 & [C2mim] [BF4] & 10 & 197.97 & 596.23 & $300-390$ & $\begin{array}{c}1.74 \cdot 10^{-1} \\
-1.83 \cdot 10^{-1}\end{array}$ & -8.04 & 8.04 & 8.44 \\
\hline 14 & [C6mim] [BF4] & 10 & 254.08 & 689.98 & $293-353$ & $\begin{array}{c}1.60 \cdot 10^{-1} \\
-1.65 \cdot 10^{-1}\end{array}$ & 3.06 & 3.82 & 5.67 \\
\hline 15 & [C8mim] [BF4] & 3 & 282.14 & 736.99 & $294.2-334.4$ & $\begin{array}{c}1.54 \cdot 10^{-1} \\
-1.57 \cdot 10^{-1}\end{array}$ & -4.58 & 4.58 & 4.97 \\
\hline 16 & [C2mim] [bti] & 9 & 391.32 & 1249.31 & $273.15-353.15$ & $\begin{array}{c}1.34 \cdot 10^{-1} \\
-1.37 \cdot 10^{-1}\end{array}$ & 12.97 & 12.97 & 14 \\
\hline 17 & [C6mim] [bti] & 9 & 447.43 & 1292.78 & $273.15-353.15$ & $\begin{array}{c}1.25 \cdot 10^{-1} \\
-1.28 \cdot 10^{-1}\end{array}$ & 3.35 & 3.35 & 4.54 \\
\hline 18 & [C10mim] [bti] & 7 & 503.53 & 1279.63 & $293-353$ & $\begin{array}{c}1.18 \cdot 10^{-1} \\
-1.21 \cdot 10^{-1}\end{array}$ & -8.12 & 8.12 & 8.97 \\
\hline 19 & [C4mim] [PF6] & 7 & 284.19 & 719.39 & $293-353$ & $\begin{array}{c}1.51 \cdot 10^{-1} \\
-1.56 \cdot 10^{-1}\end{array}$ & 6.17 & 6.17 & 7.13 \\
\hline 20 & [C6mim] [PF6] & 3 & 312.24 & 764.89 & $294.1-335.2$ & $\begin{array}{c}1.45 \cdot 10^{-1} \\
-1.48 \cdot 10^{-1}\end{array}$ & 1.15 & 1.15 & 2 \\
\hline 21 & [M1.2P3im] [bti] & 10 & 419.37 & 1269.71 & $300-390$ & $\begin{array}{c}1.27 \cdot 10^{-1} \\
-1.31 \cdot 10^{-1} \\
\end{array}$ & 0.7 & 0.7 & 1.12 \\
\hline 22 & [dbim] [bti] & 9 & 461.45 & 1305.03 & $273.15-353.15$ & $\begin{array}{c}1.23 \cdot 10^{-1} \\
-1.26 \cdot 10^{-1}\end{array}$ & 4.85 & 4.85 & 5.76 \\
\hline 23 & [bpy] [BF4] & 3 & 223.02 & 597.61 & $294.2-334.3$ & $\begin{array}{c}1.69 \cdot 10^{-1} \\
-1.73 \cdot 10^{-1}\end{array}$ & 0.31 & 0.5 & 0.9 \\
\hline 24 & [hpy] [BF4] & 3 & 251.08 & 644.98 & $294.2-334.3$ & $\begin{array}{c}1.61 \cdot 10^{-1} \\
-1.64 \cdot 10^{-1} \\
\end{array}$ & -0.13 & 0.53 & 0.59 \\
\hline 25 & [opy] [BF4] & 3 & 279.13 & 692.34 & $294.2-334.3$ & $\begin{array}{c}1.53 \cdot 10^{-1} \\
-1.56 \cdot 10^{-1}\end{array}$ & -3.18 & 3.18 & 3.57 \\
\hline 26 & [P14] [bti] & 4 & 422.42 & 1093.10 & $293-323$ & $\begin{array}{c}1.28 \cdot 10^{-1} \\
-1.29 \cdot 10^{-1} \\
\end{array}$ & 2.68 & 2.68 & 2.89 \\
\hline 27 & [N1888] [bti] & 9 & 648.86 & 1295.25 & $273.15-353.15$ & $\begin{array}{c}1.17 \cdot 10^{-1} \\
-1.20 \cdot 10^{-1}\end{array}$ & -6.86 & 6.86 & 8.74 \\
\hline 28 & [P666(14)] [bti] & 7 & 764.02 & 1471.59 & $285.65-333.55$ & $\begin{array}{c}1.34 \cdot 10^{-1} \\
-1.36 \cdot 10^{-1}\end{array}$ & -1.01 & 1.12 & 2.36 \\
\hline
\end{tabular}

Fuente: elaboración propia 
utilizados en el proceso de correlación y generalización, tal como se muestra en la tabla 4. En total, 50 datos experimentales (que representan 10 líquidos iónicos) son utilizados en este proceso. En la tabla 4 se ilustran las desviaciones obtenidas para cada líquido iónico y los rangos de temperatura, conductividad térmica y el número de datos. Los resultados muestran que las desviaciones absolutas promedio son de $3.48 \%(\mathrm{MD} \lambda=5.13 \%)$, mientras que la desviación relativa promedio es de $-0.79 \%$.

\section{Comparación de los resultados con otros trabajos desarrollados en la literatura}

El modelo generalizado de conductividad térmica se compara con otros desarrollados en la literatura y los resultados se muestran en la figura 3 . Para realizar la comparación, se utilizan los líquidos iónicos comunes a partir de la revisión de diferentes trabajos de la literatura y se estiman, para cada líquido iónico y para cada rango de temperatura, las conductividades térmicas. Los líquidos

Tabla 3. Ecuaciones matemáticas del modelo generalizado de conductividad térmica

\begin{tabular}{|c|l|}
\hline Descripción & \multicolumn{1}{|c|}{ Ecuaciones matemáticas } \\
\hline $\begin{array}{c}\text { Expresión matemática } \\
\text { del modelo empírico }\end{array}$ & $\lambda=\frac{\mathrm{a}+\mathrm{bT}_{\mathrm{r}}}{\left(\mathrm{c}+\mathrm{T}_{\mathrm{r}}\right)^{\mathrm{d}}}$ \\
\hline Valores de las constantes & $\mathrm{a}=5.67 \cdot 10^{-7} \cdot \mathrm{M}^{2}-6.62 \cdot 10^{-4} \cdot \mathrm{M}+3.35 \cdot 10^{-1}$ \\
de generalización & $\mathrm{b}=-7.70 \cdot 10^{-2}, \mathrm{c}=8.78 \cdot 10^{-2}$, \\
& $\mathrm{d}=-5.35 \cdot 10^{-2}$
\end{tabular}

Fuente: elaboración propia

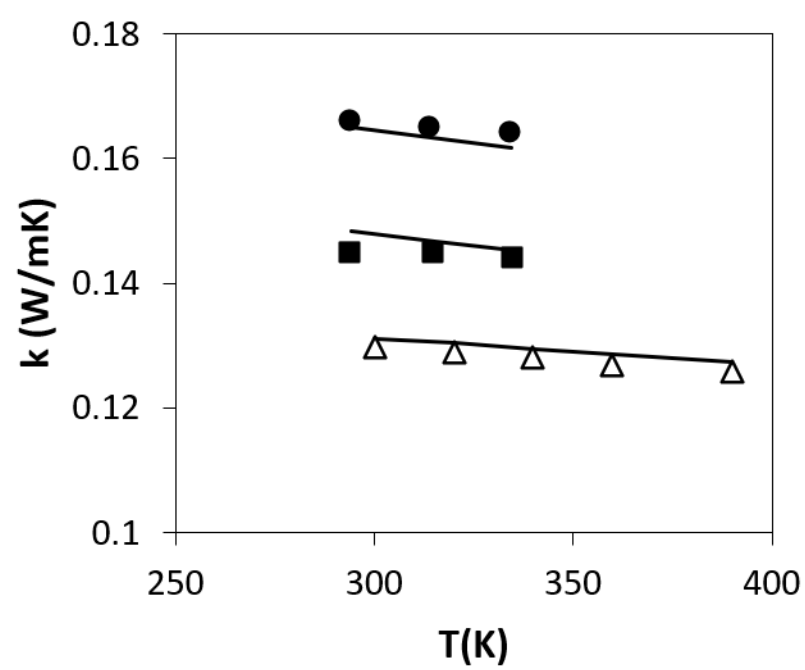

$\bullet[\mathrm{C} 6 \mathrm{mim}][\mathrm{BF} 4] \quad \boldsymbol{a}[\mathrm{C} 6 \mathrm{mim}][\mathrm{PF} 6]$

$\Delta[\mathrm{M} 1,2 \mathrm{P} 3 \mathrm{im}][\mathrm{bti}]$

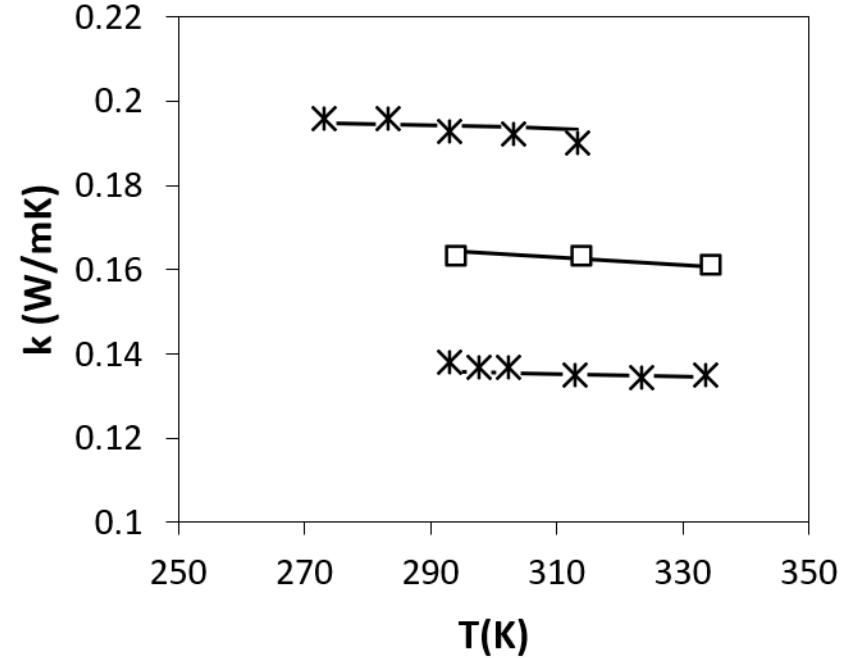

* [C2mim] [tcc] $\square[\mathrm{hpy}][\mathrm{BF} 4] \quad *[\mathrm{P} 666(14)][\mathrm{bti}]$

Figura 2. Comparación entre las conductividades térmicas calculadas y experimentales durante el proceso de correlación. La línea sólida corresponde al modelo generalizado y los círculos, cuadrados y rectángulos corresponden a datos experimentales.

Fuente: elaboración propia. 
Tabla 4. Líquidos iónicos utilizados en el proceso de predicción junto con las desviaciones obtenidas en la generalización

\begin{tabular}{|c|c|c|c|c|c|c|c|c|c|}
\hline No. & Líquido iónico & $\mathbf{N}$ & $M(\mathrm{~g} / \mathrm{mol})$ & $\mathbf{T}_{\mathbf{c}}(\mathbf{K})$ & $\Delta \mathrm{T}(\mathrm{K})$ & $\Delta \lambda(\mathbf{W} / \mathbf{m K})$ & $\mathrm{AD} \lambda$ & $\mathrm{AAD} \lambda$ & $\mathrm{MD} \lambda$ \\
\hline 1 & [C8mim] [B(CN)4] & 8 & 276.20 & 1144.01 & $283.15-353.15$ & $\begin{array}{c}1.64 \cdot 10^{-1} \\
-1.67 \cdot 10^{-1}\end{array}$ & 2.1 & 3.59 & 7.96 \\
\hline 2 & [C2mim] [DEP] & 2 & 264.26 & 877.19 & $313.15-375$ & $\begin{array}{c}1.61 \cdot 10^{-1} \\
-1.65 \cdot 10^{-1}\end{array}$ & -10.42 & 10.42 & 15.31 \\
\hline 3 & [C4mim] [tcc] & 8 & 229.29 & 1185.07 & $283.15-353.15$ & $\begin{array}{c}1.81 \cdot 10^{-1} \\
-1.84 \cdot 10^{-1} \\
\end{array}$ & 2.32 & 2.32 & 3.63 \\
\hline 4 & [C4mim] [BF4] & 3 & 226.03 & 643.18 & $294.7-334.9$ & $\begin{array}{c}1.70 \cdot 10^{-1} \\
-1.74 \cdot 10^{-1} \\
\end{array}$ & 1.68 & 1.68 & 2.57 \\
\hline 5 & [C4mim] [bti] & 7 & 419.37 & 1269.93 & $293-353$ & $\begin{array}{c}1.29 \cdot 10^{-1} \\
-1.31 \cdot 10^{-1}\end{array}$ & 3.16 & 3.16 & 4.03 \\
\hline 6 & [C8mim] [bti] & 7 & 475.48 & 1317.82 & $293-353$ & $\begin{array}{c}1.21 \cdot 10^{-1} \\
-1.24 \cdot 10^{-1}\end{array}$ & -3.62 & 3.62 & 4.27 \\
\hline 7 & [C8mim] [PF6] & 3 & 340.30 & 810.85 & $295.1-335.2$ & $\begin{array}{c}1.39 \cdot 10^{-1} \\
-1.42 \cdot 10^{-1} \\
\end{array}$ & -3.38 & 3.38 & 3.93 \\
\hline 8 & [bdmim] [bti] & 1 & 433.40 & 1281.11 & $311.1-311.1$ & $\begin{array}{c}1.28 \cdot 10^{-1} \\
-1.28 \cdot 10^{-1} \\
\end{array}$ & -1.48 & 1.48 & 1.48 \\
\hline 9 & [hmdmapy] [bti] & 2 & 501.52 & 1236.28 & $300-375$ & $\begin{array}{c}1.17 \cdot 10^{-1} \\
-1.20 \cdot 10^{-1} \\
\end{array}$ & -1.72 & 1.72 & 3.12 \\
\hline 10 & [P666(14)] [TMPPh] & 9 & 773.29 & 1729.59 & $282.47-353.62$ & $\begin{array}{c}1.37 \cdot 10^{-1} \\
-1.39 \cdot 10^{-1}\end{array}$ & 3.47 & 3.47 & 5.04 \\
\hline
\end{tabular}

Fuente: elaboración propia.

iónicos comunes son: [C6mim] [bti], [C8mim] [bti], [C2mim] [Ac], [C2mim] [tcc], [P14] [bti], [C6mim] [BF4], [C8mim] [BF4], [C4mim] [PF6], [C2mim] [bti] y el rango de temperaturas varía entre $278-343 \mathrm{~K}(P=1 \mathrm{~atm})$. Las referencias consultadas para realizar los diferentes estimativos son: [C6mim] [bti], [C8mim] [bti] (Ge et al. 2007), [C2mim] [Ac], [C2mim] [tcc] (Fröba et al., 2010), [P14] [bti] (Hezave et al. 2012), [C6mim] [BF4], [C8mim] [BF4] (Tomida et al., 2012), [C4mim] [PF6], [C2mim] [bti] (Frez et al. 2006), [C6mim] [bti], [C8mim] [bti], [C2 $\mathrm{mim}]$ [Ac], [C2 $\mathrm{mim}]$ [tcc], [P14] [bti], [C6mim] [BF4], [C8mim] [BF4], [C4mim] [PF6], [C2mim] [bti] (Cardona y Valderrama (2020a)).

Los modelos para comparar corresponden a empíricos (Fröba et al., 2010; Ge et al. 2007; Tomida et al., 2012), basados en redes neuronales (Hezave et al. 2012), modelo teórico desarrollado por Frez et al. (2006) y modelación basada en el concepto de similitud geométrica utilizando la ecuación de estado de Patel-Teja-Valderrama (Cardona y Valderrama (2020a)).
De la figura 3 se infiere que el modelo generalizado desarrollado en este trabajo tiene la menor AAD $\lambda$ cuando es comparado con Fröba et al., 2010, Tomida et al., 2012, Frez et al. (2006) y Cardona y Valderrama (2020a); sin embargo, los modelos de Ge et al. (2007) y Hezave et al., 2012 tienen los menores valores de AAD $\lambda$. Estos dos últimos modelos son utilizados para un número reducido de líquidos iónicos, no queda claro qué sustancias son utilizadas para la correlación y predicción y no se reportan parámetros estadísticos como desviaciones absolutas, relativas y valores máximos de desviación, en comparación con este trabajo cuyo modelo generalizado es simple de estimar y todas las desviaciones y fluidos utilizados en la correlación y predicción se encuentran de manera expedita. Por otra parte, los altos valores de desviación estándar (ver barras de error de la figura 3) del modelo generalizado son debidos al proceso de generalización, que introduce errores en la aproximación para cada líquido iónico; no obstante, todas estas desviaciones se encuentran dentro de la incertidumbre experimental. 


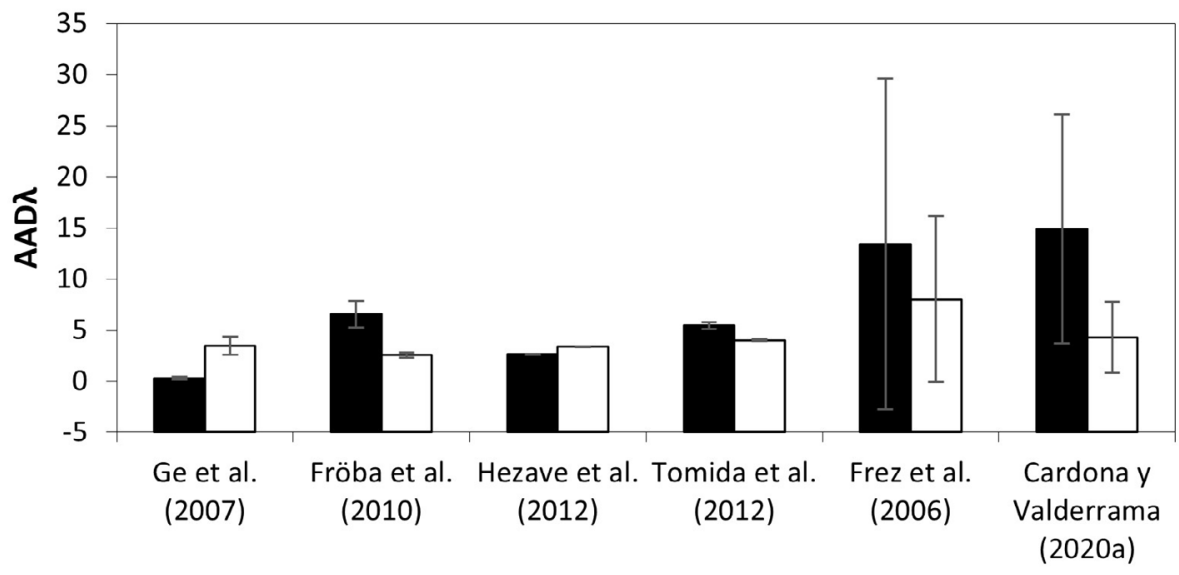

Modelos

Modelos Literatura $\quad \square$ Modelo desarrollado en este trabajo

Figura 3. Comparación de la AAD $\lambda$ entre diferentes modelos de conductividad térmica. Las barras de error corresponden a la desviación estándar de la AAD $\lambda$.

Fuente: elaboración propia

Es importante aclarar que el modelo de Chen et al. (2020) no se encuentra generalizado y es utilizado para realizar predicciones de la conductividad térmica en refrigerantes, con desviaciones absolutas que varían entre $0.37 \%$ y $3.65 \%$, en comparación con este trabajo donde este modelo ha sido generalizado para predecir la conductividad térmica de líquidos iónicos con desviaciones que varían entre 3.48 \% (proceso de predicción) y $4.72 \%$ (proceso de generalización), siendo este el aporte científico de este trabajo frente a la literatura, ya que son pocos los modelos que se encuentran generalizados.

\section{Resultados de mezclas binarias conformadas por agua+líquido iónico}

El modelo se extiende a mezclas binarias utilizando el modelo propuesto por Huang et al. (2015), en particular para mezclas formadas por (1)agua+(2)líquido iónico, tal como se muestra en la tabla 5 y figura 4 . En total, tres mezclas binarias son analizadas en este trabajo, representando 21 datos experimentales recolectados por la NIST-LIs.
Es importante aclarar que las soluciones acuosas son ampliamente utilizadas en procesos fisicoquímicos y biológicos (Poling et al., 2001). Los rangos de temperatura varían entre $293 \mathrm{~K}$ y $313 \mathrm{~K}$ a 1 atm.

Para tal fin, se estiman para el agua los parámetros $a, b, c$ y $d$ del modelo de conductividad térmica (ecuación 1), utilizando los datos experimentales reportados como recomendados y aceptados por la base de datos de la DIPPR (Daubert, 1998), ya que el agua exhibe un comportamiento diferente al resto de fluidos (Fang et al., 2019). Los valores de los parámetros para el agua son: $a=$ $5.93 \cdot 10^{-1}, b=5.74 \cdot 10^{-2}, c=9.01 \cdot 10^{-2}, d=-4.83 \cdot 10^{-}$

${ }^{2}$ en un rango de temperatura que varía entre 280 y $313 \mathrm{~K}$ (1 atm). Posteriormente, se estima para cada mezcla reportada en la tabla 5 la conductividad térmica usando las ecuaciones 1,5 y 6 . Es importante aclarar que la regla de mezcla representada por estas ecuaciones requiere la estimación de la conductividad térmica de los componentes puros (agua y líquido iónico).

Al utilizar la regla de mezcla de Huang et al. (2015) sin parámetro de interacción, $(\pi=0$, ver ecuaciones 5 y 6), se tiene una desviación absoluta 
relativa promedio $\left(\mathrm{AAD} \lambda_{\mathrm{m}}\right)$ de $65.11 \%\left(\mathrm{MD} \lambda_{\mathrm{m}}=\right.$ $156 \%$ ); sin embargo, al estimar $\pi$ a partir de datos experimentales y minimizando la ecuación $2(\pi$ $\neq 0)$, la $\mathrm{AAD} \lambda_{\mathrm{m}}$ es de $0.77 \%\left(\mathrm{MD} \lambda_{\mathrm{m}}=9.22 \%\right)$, la desviación relativa promedio tiene un valor de 0.25 $\%$. El parámetro de interacción binaria $(\pi)$ varía entre -0.4522 y -0.03758 (ver tabla 5, columna 7).

En la figura 4 se muestra las desviaciones absolutas $\left(\mathrm{AAD} \lambda_{\mathrm{m}}\right)$ y los valores máximos de desviación $\left(M D \lambda_{m}\right)$ de las tres mezclas estudiadas en este trabajo. En particular, la mezcla 3 ((1)agua+(2) [C4mim][TfO]) tiene el valor más alto de desviación absoluta (MD $\left.\lambda_{\mathrm{m}}=9.22 \%\right)$, que corresponde a una composición $x_{1}=0$ (1: agua, 2: líquido iónico) y se debe al proceso de generalización del componente puro. Para las demás mezclas ((1) agua $+(2)[\mathrm{C} 2 \mathrm{mim}][\mathrm{ESO} 4]$ y (1)agua+(2)[C2 mim] [DEP]) las desviaciones absolutas son inferiores de $0.88 \%$ con máximos de desviación inferiores de
$2.43 \%$. Todo lo anterior se ve reflejado en la desviación estándar, que para la mezcla 3 tiene una mayor dispersión de los datos, por lo tanto su desviación estándar es mayor.

En conclusión, al utilizar el modelo de Huang et al. (2015) es necesario estimar para cada mezcla el parámetro de interacción binaria, $\pi$. Estos resultados son similares a los presentados por Huang et al. (2015), quienes reportan valores de $\operatorname{AAD} \lambda_{\mathrm{m}}$ de $1.90 \%\left(\mathrm{MD} \lambda_{\mathrm{m}}=7.57 \%\right)$.

\section{Conclusiones}

Del presente trabajo se pueden extraer las siguientes conclusiones:

1) Se generaliza un modelo simple y empírico como alternativa para describir las conductividades térmicas de líquidos iónicos. Los cuatro

Tabla 5. Intervalos de temperatura, presión y desviaciones utilizando la regla de mezcla de Huang et al. (2015) con coeficiente de interacción estimado a partir de datos experimentales.

\begin{tabular}{|c|l|c|c|c|c|c|c|c|c|}
\hline No. & \multicolumn{1}{|c|}{ Mezcla } & $\mathbf{N}$ & $\boldsymbol{\Delta} \mathbf{T}(\mathbf{K})$ & $\boldsymbol{\Delta} \boldsymbol{\lambda} \mathbf{( W / m K})$ & $\boldsymbol{\Delta} \mathbf{x}_{\mathbf{1}}$ & $\boldsymbol{\pi}$ & $\mathbf{A D} \boldsymbol{\lambda}_{\mathbf{m}}$ & $\mathbf{A A D} \boldsymbol{\lambda}_{\mathbf{m}}$ & $\mathbf{M D} \boldsymbol{\lambda}_{\mathbf{m}}$ \\
\hline 1 & (1)agua+(2)[C2mim][ESO4] & 8 & 293 & $0.181-0.607$ & $0-1$ & $-0.34--0.038$ & -0.25 & 0.88 & 2.43 \\
\hline 2 & (1)agua+(2)[C2mim][DEP] & 5 & 313.15 & $0.175-0.234$ & $0.2-1$ & $-0.129--0.038$ & 0.01 & 0.12 & 0.28 \\
\hline 3 & (1)agua+(2)[C4mim][TfO] & 8 & 293 & $0.147-0.607$ & $0-1$ & $-0.452--0.046$ & 1.01 & 1.32 & 9.22 \\
\hline
\end{tabular}

Fuente: elaboración propia.

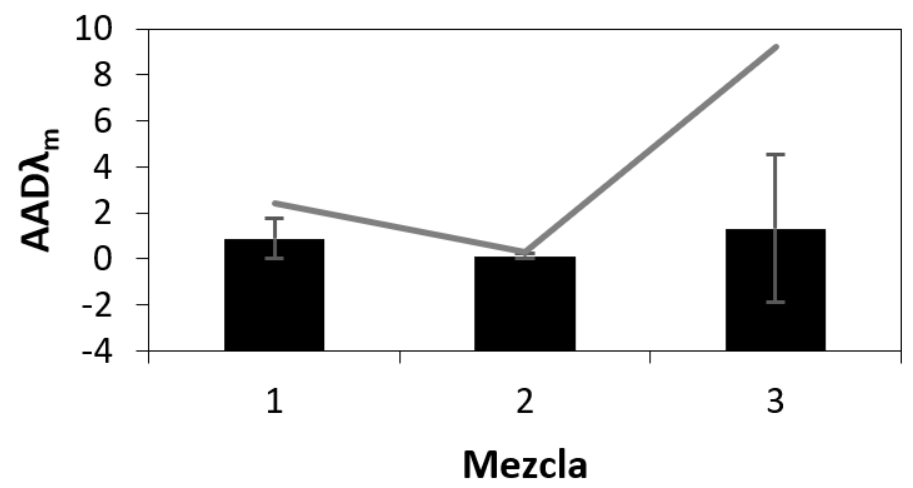

Mezcla 1. (1)agua+(2)[C2mim][ESO4] Mezcla 2. (1)agua $+(2)[\mathrm{C} 2 \mathrm{mim}][\mathrm{DEP}]$ Mezcla 3. (1)agua+(2)[C4mim][TfO]

Figura 4. Desviaciones absolutas relativas promedio de cada mezcla utilizando la ecuación de Huang et al. (2015). Las barras de error indican la desviación estándar de la $A A D \lambda_{m}$ y la línea gris corresponde a los valores de $M D \lambda_{m}$.

Fuente: elaboración propia. 
parámetros del modelo $(a, b, c$ y $d)$ son generalizados utilizando expresiones matemáticas simples, donde el parámetro a es una función cuadrática en términos del peso molecular y $b, c$ y $d$ son constantes para todos los líquidos iónicos. El rango de temperatura varía entre $273 \mathrm{~K}$ y $390 \mathrm{~K}$ a 1 atm;

2) Las desviaciones absolutas relativas promedio (AAD $\lambda$ ) durante el proceso de generalización y predicción son de $4.72 \%$ y $3.48 \%$ con valores máximos de $5.96 \%$.

3) El modelo generalizado es comparado con otros desarrollados en la literatura y los resultados estadísticos muestran que este proporciona resultados aceptables.

4) El modelo se extiende a mezclas, utilizando la regla de mezcla simple propuesta por Huang et al. (2015) y las desviaciones absolutas relativas promedio son de $0.77 \%$.

5) El principal aporte de este trabajo frente otros propuestos en la literatura es el desarrollo de un modelo generalizado simple de estimar y la realización de predicciones aceptables de la conductividad térmica de líquidos iónicos; asimismo, se reportan todas las desviaciones (absolutas, relativas y valores máximos) con el propósito de mostrar la bondad del modelo.

\section{Agradecimientos}

El autor agradece a la Universidad Católica Luis Amigó sede Medellín (Colombia) por el apoyo en la realización de la presente investigación.

\section{Referencias}

Asensio-Delgado, S., Jovell, D., Zarca, G., Urtiaga, A., Llovell, F. (2020). Thermodynamic and process modeling of the recovery of R410A compounds with ionic liquids. International Journal of Refrigeration, 118, 365-375. https://doi.org/10.1016/j. ijrefrig.2020.04.013

Akhgar, A., Toghraie, D., Sina, N., Afrand, M. (2019). Developing dissimilar artificial neural networks (ANNs) to prediction the thermal conductivity of MWCNT-TiO 2 /Water-ethylene glycol hybrid nanofluid. Powder Technology, 355, 602-610. https:// doi.org/10.1016/j.powtec.2019.07.086

Alvarez-Guerra, M., Luis, P., Irabien, A. (2011). Modelo de contribución de grupos para la estimación de la ecotoxicidad de líquidos iónicos. Afinidad, 68(551), 20-24

Bloxham, J. C., Redd, M. E., Giles, N. F., Knotts IV, T. A., Wilding, W. V. (2021). Proper use of the DIPPR 801 database for creation of models, methods, and processes. Journal of Chemical \& Engineering Data, 66(1), 3-10. https://doi.org/10.1021/acs. jced.0c00641

Bonyadi, M., Rostami, M. (2017). A new viscosity model based on Soave-Redlich-Kwong equation of state. Fluid Phase Equilibria, 451, 40-47. https://doi. org/10.1016/j.fluid.2017.07.009

Bhunia, P., Ghangrekar, M. M. (2008). Analysis, evaluation, and optimization of kinetic parameters for performance appraisal and design of UASB reactors. Bioresource Technology, 99(7), 2132-2140. https://doi.org/10.1016/j.biortech.2007.05.053

Burke, J. A. (2006). Two mathematical programming models of cheese manufacture. Journal of Dairy Science, 89(2), 799-809. https://doi.org/10.3168/ jds.S0022-0302(06)72142-7

Cardona, L. F. (2016). Nueva correlación generalizada para estimar la presión de vapor. Revista Científica, 25(2), 280-289. https://doi.org/10.14483//udistrital. jour.RC.2016.25.a11

Cardona, L. F., Forero, L. A., Velásquez, J. A. (2019). Correlation and prediction of thermal conductivity using the Redlich-Kwong cubic equation of state and the geometric similitude concept for pure substances and mixtures. Industrial \& Engineering Chemistry Research, 58(51), 23417-23437. https://doi. org/10.1021/acs.iecr.9b04974

Cardona, L. F., Valderrama, J. O. (2020a). Physical and transport properties of ionic liquids using the geometric similitude concept and a cubic equation of state. Part 1: Thermal conductivity and speed of sound of pure substances. Journal of Molecular Liquids, 315. https://doi.org/10.1016/j. molliq.2020.113681 
Cardona, L. F., Valderrama, J. O. (2020b). Surface tension of mixtures containing ionic liquids based on an equation of state and on the geometric similitude concept. Ionics, 26(12), 6095-6118. https://doi. org/10.1007/s11581-020-03697-0

Castillo, F. (2015). Líquidos iónicos: métodos de síntesis y aplicaciones. ConCiencia Tecnológica, 49, 52-56

Chen, Z., Akbari, M., Forouharmanesh, F., Keshani, M., Akbari, M., Afrand, M., Karimipour, A. (2020). A new correlation for predicting the thermal conductivity of liquid refrigerants. Journal of Thermal Analysis and Calorimetry, 143, 795-800. https:// doi.org/10.1007/s10973-019-09238-w

Daubert, T. E. (1998). Evaluated equation forms for correlating thermodynamic and transport properties with temperature. Industrial \& Engineering Chemistry Research, 37(8), 3260-3267. https://doi. org/10.1021/ie9708687

Díaz-Parra, O., Vera-López, E. (2018). Simulación de áreas de alta consecuencia para gasoductos. Científica, 31(1), 32-44. https://doi. org/10.14483/23448350.12513

Dong, Q., Kazakov, A., Muzny, C., Chirico, R., Widegren, J., Diky, V., Magee, J., Marsh, K., Frenkel, M. (2007). ILThermo: A free-access web database for thermodynamic properties of ionic liquids. Journal of Chemical \& Engineering Data, 52(4), 1151-1159. https://doi.org/10.1021/je700171f

Fang, H., Ni, K., Wu, J., Li, J., Huang, L., Reible, D. (2019). The effects of hydrogen bonding on the shear viscosity of liquid water. International Journal of Sediment Research, 34(1), 8-13. https://doi. org/10.1016/j.ijsrc.2018.10.008

Fröba, A. P., Rausch, M. H., Krzeminski, K., Assenbaum, D., Wasserscheid, P., Leipertz, A. (2010). Thermal conductivity of ionic liquids: Measurement and prediction. International Journal of Thermophysics, 31(11), 20592077. https://doi.org/10.1007/s10765-010-0889-3

Frez, C., Diebold, G. J., Tran, C. D., Yu, S. (2006). Determination of thermal diffusivities, thermal conductivities, and sound speeds of room-temperature ionic liquids by the transient grating technique. Journal of Chemical \& Engineering Data, 51(4), 1250-1255. https://doi.org/10.1021/je0600092
Gardas, R. L., Coutinho, J. A. (2009). Group contribution methods for the prediction of thermophysical and transport properties of ionic liquids. AIChE Journal, 55(5), 1274-1290. https://doi.org/10.1002/ aic. 11737

Ge, R., Hardacre, C., Nancarrow, P., Rooney, D. W. (2007). Thermal conductivities of ionic liquids over the temperature range from $293 \mathrm{~K}$ to $353 \mathrm{~K}$. Journal of Chemical \& Engineering Data, 52(5), 1819-1823. https://doi.org/10.1021/je700176d

Godsen, S. (2002). Optimization analysis of projectile motion using spreadsheets. The Physics Teacher, 40(9), 523-525. https://doi.org/10.1119/1.1534816

Hezave, A. Z., Raeissi, S., Lashkarbolooki, M. (2012). Estimation of thermal conductivity of ionic liquids using a perceptron neural network. Industrial \& Engineering Chemistry Research, 51(29), 9886-9893. https://doi.org/10.1021/ie202681b

Hopp, M., Gross, J. (2019). Thermal conductivity from entropy scaling: A group-contribution method. Industrial \& Engineering Chemistry Research, 58(44), 20441-20449. https://doi.org/10.1021/acs. iecr.9b04289

Huang, H.-J., Lin, W., Ramaswamy, S., Tschirner, U. (2009). Process modeling of comprehensive integrated forest biorefinery: An integrated approach. Applied Biochemistry and Biotechnology, 154(1), 26-37. https://doi.org/10.1007/s12010-008-8478-7

Huang, Y., Zhang, X., Zhao, Y., Zeng, S., Dong, H., Zhang, S. (2015). New models for predicting thermophysical properties of ionic liquid mixtures. Physical Chemistry Chemical Physics, 17(40), 26918-26929. https://doi.org/10.1039/C5CP03446A

Iglesias, O. A., Pastor, J., Henseler, A., Bertolini, R., Soto, J. D., Paniagua, C. N. (2004). Spreadsheet use in conceptual design of chemical processes. International Journal of Engineering Education, 20(6), 999-1004

Kazakov A., Magee J.W., Chirico R.D., Paulechka E., Diky V., Muzny C.D., Kroenlein K., Frenkel M. (s. f.). NIST Standard Reference Database 147: NIST Ionic Liquids Database (ILThermo), Version 2.0. Gaithersburg, MD: National Institute of Standards and Technology. http://ilthermo.boulder.nist.gov 
Kemmer, G., Keller, S. (2010). Nonlinear least-squares data fitting in Excel spreadsheets. Nature Protocols, 5(2), 267-281. https://doi.org/10.1038/ nprot.2009.182

Lasdon, L. S., Waren, A. D., Jain, A., Ratner, M. (1978). Design and testing of a generalized reduced gradient code for nonlinear programming. ACM Transactions on Mathematical Software, 4(1), 34-50. https://doi.org/10.1145/355769.355773

Minea, A. A. (2020). Overview of ionic liquids as candidates for new heat transfer fluids. International Journal of Thermophysics, 41. https://doi.org/10.1007/ s10765-020-02727-3

Poling, B. E., Prausnitz, J. M., O'Connell, J. P. (2001). Properties of Gases and Liquids. Nueva York: McGraw-Hill Education

Ranjbarzadeh, R., Moradikazerouni, A., Bakhtiari, R., Asadi, A., Afrand, M. (2019). An experimental study on stability and thermal conductivity of water/silica nanofluid: Eco-friendly production of nanoparticles. Journal of Cleaner Production, 206, 1089-1100. https://doi.org/10.1016/j.jclepro.2018.09.205

Rivas, A., Gómez-Acebo, T., Ramos, J. C. (2006). The application of spreadsheets to the analysis and optimization of systems and processes in the teaching of hydraulic and thermal engineering. Computer Applications in Engineering Education, 14(4), 256268. https://doi.org/10.1002/cae.20085
Tomassetti, S., Coccia, G., Pierantozzi, M., Di Nicola, G. (2020). Correlations for liquid thermal conductivity of low GWP refrigerants in the reduced temperature range 0.4 to 0.9 from saturation line to $70 \mathrm{MPa}$. International Journal of Refrigeration, 117, 358-368. https://doi.org/10.1016/j.jirefrig.2020.05.004

Tomida, D., Kenmochi, S., Tsukada, T., Qiao, K., Bao, Q., Yokoyama, C. (2012). Viscosity and thermal conductivity of 1-hexyl-3-methylimidazolium tetrafluoroborate and 1-octyl-3-methylimidazolium tetrafluoroborate at pressures up to $20 \mathrm{MPa}$. International Journal of Thermophysics, 33(6), 959-969. https://doi.org/10.1007/s10765-012-1233-x

Valderrama, J. O., Álvarez, V. H. (2005). Correct way of reporting results when modelling supercritical phase equilibria using equations of state. The Canadian Journal of Chemical Engineering, 83(3), 578-581. https://doi.org/10.1002/cjce.5450830323

Valderrama, J. O., Forero, L. A., Rojas, R. E. (2019a). Critical properties of metal-containing ionic liquids. Industrial \& Engineering Chemistry Research, 58(17), 7332-7340. https://doi.org/10.1021/ acs.iecr.9b00279

Valderrama, J. O., Cardona, L. F., Rojas, R. E. (2019b). Correlation of ionic liquid viscosity using Valderrama-Patel-Teja cubic equation of state and the geometric similitude concept. Part II: Binary mixtures of ionic liquids. Fluid Phase Equilibria, 497, 178194. https://doi.org/10.1016/j.fluid.2019.04.034 